\section{Diagnostic challenges in non-small-cell lung cancer: an integrated medicine approach}

\author{
Ravi Salgia*
}

\begin{abstract}
The discovery of diverse driver mutations in lung cancer has heralded a new era of personalized medicine in thoracic oncology, with targeted therapies approved for specific subgroups of patients. The increasing number of patient subgroups that may respond to targeted therapy has resulted in a greater reliance upon effective and increasingly complex diagnostics, which must be interpreted in an interactive multidisciplinary forum. This review discusses the molecular diagnostics available and under development for established and emerging targets, and how these may be integrated into current treatment algorithms. The roles of the pulmonologist, interventional radiologist, thoracic surgeon and molecular pathologist are discussed, and their interactions with the medical oncologist, and/or thoracic surgeon and radiation oncologist in making individual treatment decisions.
\end{abstract}

Lung cancer is the most common global cause of cancer-related death in men, and second only to breast cancer in women [1]. There are over 1.5 million people diagnosed annually, worldwide, with an estimated 224,210 new diagnoses and 159,260 deaths in the USA alone in 2014 [2]. Nonsmall-cell lung cancer (NSCLC) accounts for $85 \%$ of all lung cancer cases, with the remainder classified as small-cell lung cancer (SCLC) [3,4]. The average 5-year survival rate after diagnosis with advanced NSCLC in the USA is low, at only $5 \%$ for stage IIIB disease, and just $1 \%$ for stage IV disease [5], with frequent metastasis to the bones, liver and brain [6,7]. These values highlight the high unmet medical need for the treatment of advanced lung cancer.

For many years the distinction between lung cancers was based on the histologic characteristics of resected tumors, which proved adequate for the selection of therapies. In NSCLC, the absence of therapeutic implications of further classification has meant that the subtyping of small tissue biopsy samples as adenocarcinoma or squamous cell carcinoma (SCC) has not received priority attention. However, new therapeutic strategies are emerging which require more detailed and specific disease subtyping. Moreover, because over $70 \%$ of patients with lung cancer present at an advanced stage [8], this need for detailed subtyping requires a new approach to the collection and analysis of non-resected, small biopsy samples and cytology, which can not only distinguish between adenocarcinoma and SCC, but also detect a growing number of molecular aberrations implicated in NSCLC pathogenesis that direct therapeutic strategies for individual patients. A large body of evidence now suggests that this personalized approach to NSCLC treatment will maximize the effectiveness of currently available and newly emerging targeted therapies.

Until the advent of personalized medicine, treatment for advanced NSCLC was limited to chemotherapy, with response rates typically $20-30 \%$ and progression-free survival (PFS) of 3-5 months following first-line chemotherapy [9-12]. Conventional chemotherapies remain the mainstay treatments in unselected NSCLC; however, the discovery of activating mutations in the EGFR gene

*Section of Hematology/Oncology, University of Chicago Medicine, Chicago, IL, USA; Tel.: + 1773702 6613;

rsalgia@medicine.bsd.uchicago.edu

\section{KEYWORDS}

- ALK inhibitor • EGFR inhibitor • integrated medicine $\bullet$ molecular diagnostics $\bullet$ molecular profile $\bullet$ multidisciplinary forum • non-small-cell lung cancer $\bullet$ personalized medicine 
heralded a new era of personalized medicine in thoracic oncology. Although not curative, currently available tyrosine kinase inhibitors (TKIs) such as erlotinib, gefitinib and afatinib target EGF receptor (EGFR) and provide dramatic tumor responses compared with conventional chemotherapy in patients with NSCLC harboring such mutations, with a response rate of $62-83 \%$, PFS of 9-13 months and improved quality of life compared with chemotherapy [1318]. Advances in the knowledge of the molecular origins of NSCLC have also led to the identification of an expanding number of molecular subtypes according to genetic abnormalities that can act as oncogenic drivers of malignant progression (Figure 1). Newer driver mutations that have been discovered provide further therapeutic options for specific patient subgroups, as has already been realized with the approval of the anaplastic lymphoma kinase (ALK) inhibitor crizotinib, for treating $A L K$-rearranged ( $A L K$ positive) NSCLC, where patients benefit from a $60 \%$ response rate, 9-month PFS and a low degree of toxicity $[19,20]$. Second-generation ALK inhibitors in advanced stages of development include ceritinib (LDK378; now US FDAapproved for the treatment of patients with ALK-positive metastatic NSCLC who have progressed on or are intolerant to crizotinib) [21] and alectinib (CH5424802) [22]. Both of these agents have also received US FDA Breakthrough Therapy designation in NSCLC [23,24].

Inhibitors targeting EGFR or ALK have demonstrated significant efficacy in the treatment of lung cancer and established or developing companion diagnostic assays readily identify patients who may benefit from these treatments. However, an increasing number of potentially targetable molecular aberrations are being identified which, although they occur in small patient subgroups, offer the opportunity for effective targeted therapy based on individual diagnosis. For instance, the BRAF inhibitor dabrafenib (approved for BRAF-V600E/K mutated melanoma) showed durable antitumor activity (overall response rate [ORR] $40 \%$ ) in patients with $B R A F \mathrm{~V} 600 \mathrm{E}$ mutationpositive pretreated NSCLC, and has received FDA Breakthrough Therapy designation for this indication [36]. A dramatic response was also reported following treatment with the BRAF inhibitor vemurafenib in a patient with $B R A F$ V600E-mutated lung adenocarcinoma [37]. Mutations in BRAF have been found in approximately 1-5\% of NSCLCs, almost exclusively in adenocarcinomas [38,39], indicating that routine screening may be justified. Other relatively rare genetic alterations detected in NSCLC (e.g., in MET [amplification], ROS1 [rearrangement] or PIK3CA [mutation]) [25,26] may offer similar opportunities for effective targeted therapy if they are identified by routine screening. Novel oncogenes that are potential candidates for future lung cancer therapies are also being identified; for example, rearrangements of the NTRK1 gene have also recently been identified in $3 \%$ of patients with lung adenocarcinoma $[27,40]$. Rearrangements of the rearranged during transfection (RET) gene have also been detected in $1.4 \%$ of adenocarcinomas, and render these tumors amenable to treatment with RET inhibitors [28]. According to the National Cancer Institute's Lung Cancer Mutation Consortium, 466/733 (64\%) of lung adenocarcinomas subjected to full genotyping (among 1007 NSCLC cases) were oncogeneaddicted disease driven by genetically defined aberrations as determined by multiplexed assays [41]. In a study by Sequist $e t$ al., tumor genotype analysis has identified driver alterations in approximately $50 \%$ to $80 \%$ of patients with NSCLC. Although the most common alterations were KRAS (24\%) or EGFR mutations (13\%), and $A L K$ rearrangements (5\%), several rarer alterations were identified [42]. These included PIK3CA (especially in squamous NSCLC), ERBB2 and BRAF mutations, all of which have candidate targeted therapies available. Genotyping distinguished multiple primary cancers from metastatic disease and steered $78(22 \%)$ of the 353 patients with advanced disease toward a genotype-directed targeted therapy [42]. As the lung cancer therapeutics field is expanding, immune therapy has also become important [43].

In the era of precision personalized medicine, the roles of the diverse oncologic specialists are evolving to meet the diverse challenges in the diagnosis and treatment of these patients with molecularly driven NSCLC. This review discusses an integrated approach, comprising the input and expertise of disciplines ranging from pulmonologists and pathologists to medical oncologists, which is required to adapt to the progression of the lung cancer treatment environment toward truly personalized therapy, and to meet the inevitable accompanying diagnostic and therapeutic challenges. 


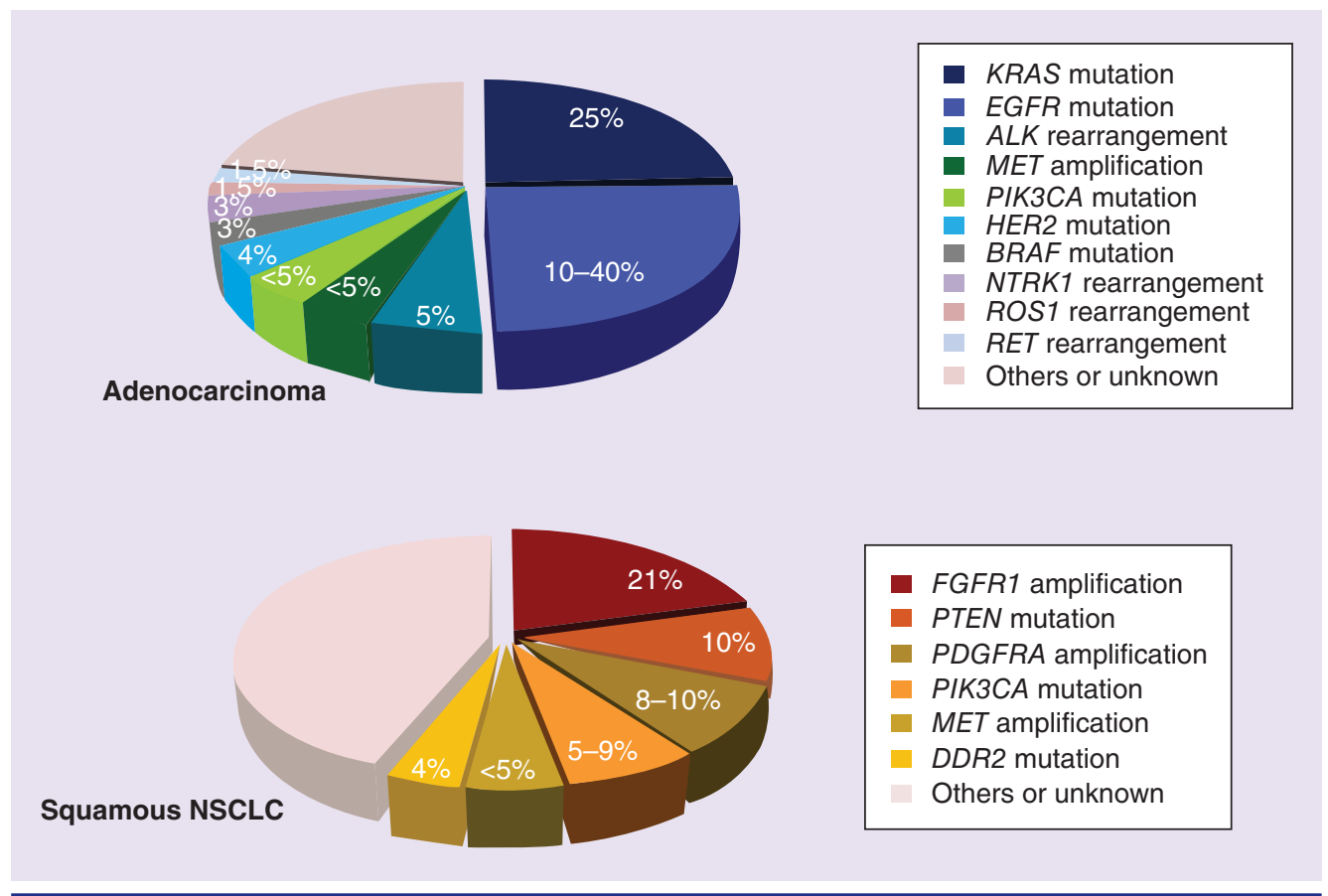

Figure 1. Clinically relevant genetic alterations in adenocarcinoma and squamous non-small-cell lung cancer.

NSCLC: Non-small-cell lung cancer.

Data taken with permission from [3,25-35].

Multidisciplinary approach to an NSCLC molecular therapeutic algorithm

\section{- Pathologic considerations}

Development of new diagnostic tools \& testing algorithms in NSCLC

Four targeted agents are now FDA and/or EMA approved for the treatment of advanced NSCLC, and current tumor screening guidelines such as those published by the National Comprehensive Cancer Network (NCCN) and the European Society for Medical Oncology (ESMO) recommend testing for activating EGFR mutations and $A L K$ rearrangements when making decisions on the selection of standard chemotherapy or targeted TKI therapy; approved companion diagnostics are available to detect these alterations $[4,44]$. However, it is likely that in the coming years, further targeted agents will be approved for the treatment of patients with lung cancer, necessitating an increasing number and diversity of molecular diagnostics. These rapid developments will require a new multidisciplinary and integrated approach to make comprehensive molecular characterization a part of routine clinical practice. Currently, guidelines are insufficient for the selection of patients for clinical trial-based evaluations of new targeted therapies in a patient population comprising an increasing number of molecular subgroups (Figure 1).

\section{Current pathologic \& molecular testing} guidelines

The standard pathologic classification of lung cancer has recently been revised by an International Association for the Study of Lung Cancer/American Thoracic Society/European Respiratory Society (IASLC/ATS/ERS) multidisciplinary panel that included oncologists, pulmonologists, pathologists, radiologists, molecular biologists and thoracic surgeons [45]. This international panel modified the previous classification of NSCLC not otherwise specified, these tumors now being classified further using a limited immunohistochemical work-up that preserves tissue for subsequent molecular testing. To this end, tissue microarray studies have now identified immunohistochemical markers that facilitate the distinction between adenocarcinoma and SCC. For example, the combination of napsin-A, TTF1, CK5/6 and p 63 differentiates adenocarcinoma from SCC, and CD141 is a potential new and highly specific marker for SCC [46].

Detailed evidence-based mutation testing recommendations for $E G F R$ mutation and $A L K$ 
rearrangement have been provided by the College of American Pathologists (CAP), IASLC and Association for Molecular Pathology (AMP) [47]. EGFR mutation testing should be used to select patients for EGFR-directed TKI therapy and $A L K$ rearrangement testing should be used to select patients for ALK TKI therapy. Testing is recommended for adenocarcinomas and mixed lung cancers with an adenocarcinoma component, regardless of grade. EGFR mutation and $A L K$ rearrangement testing are not recommended for excised specimens if there is no adenocarcinoma component (e.g., pure squamous or large cell carcinomas with no histochemical evidence of adenocarcinoma differentiation). However, this should be cautiously interpreted since there are cases where EGFR mutations are found in SCC as well as SCLC [48,49]. For limited specimens (biopsies, cytology) where adenocarcinoma components cannot be completely excluded, testing may be performed if squamous or small cell histology is shown, but clinical criteria, including young age and lack of smoking history, may be useful in selecting a subset of these samples for testing. For EGFR, any validated EGFR mutation testing method able to detect mutations in specimens with $\geq 50 \%$ cancer cell content is recommended, but the use of more sensitive tests that can detect mutations in specimens with $\geq 10 \%$ cancer cells is encouraged. The test should be able to detect all mutations reported in $\geq 1 \%$ of $E G F R$-mutated adenocarcinomas. Immunohistochemistry (IHC) for total EGFR, and EGFR copy number analysis (FISH/chromogenic in situ hybridization [CISH]) are not recommended for selection of therapy. If a specimen is from a patient with acquired resistance to EGFR inhibitors, tests should be able to detect the secondary EGFR T790M mutation in as few as $5 \%$ of cells. Validated methods include Sanger sequencing, length analysis, restriction fragment length polymorphism, real-time PCR, melting curve analysis and mass spectrometry (MS)-based genotyping. EGFR mutation testing is currently prioritized over other molecular markers.

After activating $E G F R$ mutation testing, testing for ALK is prioritized over other proposed molecular markers in lung adenocarcinoma, for which published evidence is currently insufficient to support testing guideline development $[4,47]$. For $A L K$ rearrangement testing, an ALK FISH assay using dual-labeled break-apart probes is recommended for selecting patients for ALK TKI therapy; ALK immunohistochemistry may be considered as a screening methodology to select specimens for ALK FISH testing. Reverse transcription PCR (RT-PCR) is not recommended as an alternative to FISH for patient selection, although more recent quantitative assays are able to distinguish $A L K$ rearrangements and full-length transcript expression in formalin-fixed paraffin-embedded (FFPE) samples [50]. The technique has also identified ALK deregulation in cases not identified through FISH [50]. PCR-based techniques are used to characterize secondary mutations in $A L K$ associated with acquired resistance to crizotinib [51]. However, identification of secondary mutations is not currently required for clinical management, although it may aid future decisions on the treatment of patients with $A L K$-rearranged NSCLC who have progressed on crizotinib using new ALK inhibitors under development.

Optimum diagnostic methodologies for newer genetic alterations such as $A L K$ rearrangement have not been definitively established. For example, although FISH is the FDA-approved clinical standard that detects all variants and is suitable for FFPE specimens [52,53], not all patients with NSCLC who may benefit from ALK inhibitor treatment are identified [54-56]. FISH has a number of disadvantages, being both more time-consuming and technically demanding than some other methods, with variability among observers, and scoring complications [52-53,57]. By contrast, IHC is a less time-consuming [58], relatively simple and widely available procedure [52]. Low-level differential expression of ALK protein means that careful optimization of antibody clone and detection system is required [52]. A number of antibodies are now in development, and IHC may become a standard of care diagnostic (potentially with augmentation) [53,59]. There is also some clinical evidence to suggest that diagnosis based on FISH only may less accurately predict response to crizotinib than combined FISH/IHC/RT-PCR (ORR $81 \%$ for patients diagnosed based on combined tests vs $48 \%$ for patients diagnosed by FISH only; $\mathrm{p}=0.007$ ) [60]. In addition, a recent study of ALK status in 3244 NSCLC cases by parallel FISH and IHC revealed major discordances [61]. In this study only 80 of 150 specimens were classified as $A L K$-positive by both techniques, indicating that single FISH or IHC analysis alone would have failed to detect $25 \%$ of the $A L K$-positive patients. This level of discrepancy supports combined FISH and IHC testing for determining ALK status, particularly as some patients with discordant 
results responded to crizotinib [61]. In addition, new combined parallel techniques are being investigated; for example, a novel dual IHC-in situ (brightfield break-apart) hybridization assay may provide a future more accurate determination of ALK status in NSCLC, including in heterogeneous samples [52]. The limitations of single marker-specific assays, and the identification of an increasing number of driver mutations associated with lung cancer in recent years, have led to an urgent need for strategies to improve efficiency of testing for multiple molecular abnormalities through broad clinical genotyping.

\section{Pathologic \& molecular testing: advances}

$\&$ future perspectives

In addition to the already approved companion diagnostic platforms, advances in translational genomics and proteomics have now provided the foundation for the rapid advance of the diagnostics and management of NSCLC, with a range of available diagnostic platforms either developed or under development (Figure 2). Multiplexed genotype testing with a multiplexed PCR-based clinical genotyping test (SNaPshot) has been found to be feasible within the clinical workflow, with a median turnaround time of 2.8 weeks, which includes the time necessary to acquire FFPE samples from outside hospitals [42]. This assay tests $>50$ hot-spot mutation sites in 14 key cancer genes (FISH was carried out separately for $A L K$ rearrangement), with a focus on capturing somatic events with known or putative implications for molecularly targeted therapy. This type of testing could significantly affect both early treatment decisions and those for patients with advanced or metastatic disease. Indeed, multiplex hot spot analyses, for example with Sequenom ${ }^{\circledR}$ MassARRAY platform mutation screening, is feasible on FFPE specimens and has been shown to detect genetic abnormalities in early NSCLC [62]. Multiplexed deep sequencing analysis of ALK kinase domain has also identified resistance mutations in relapsed patients following crizotinib treatment [63].

The screening and identification of mutations such as those in EGFR have been routinely carried out by direct sequencing; however, the sensitivity of direct sequencing is suboptimal for many clinical tumor samples, where detection limits

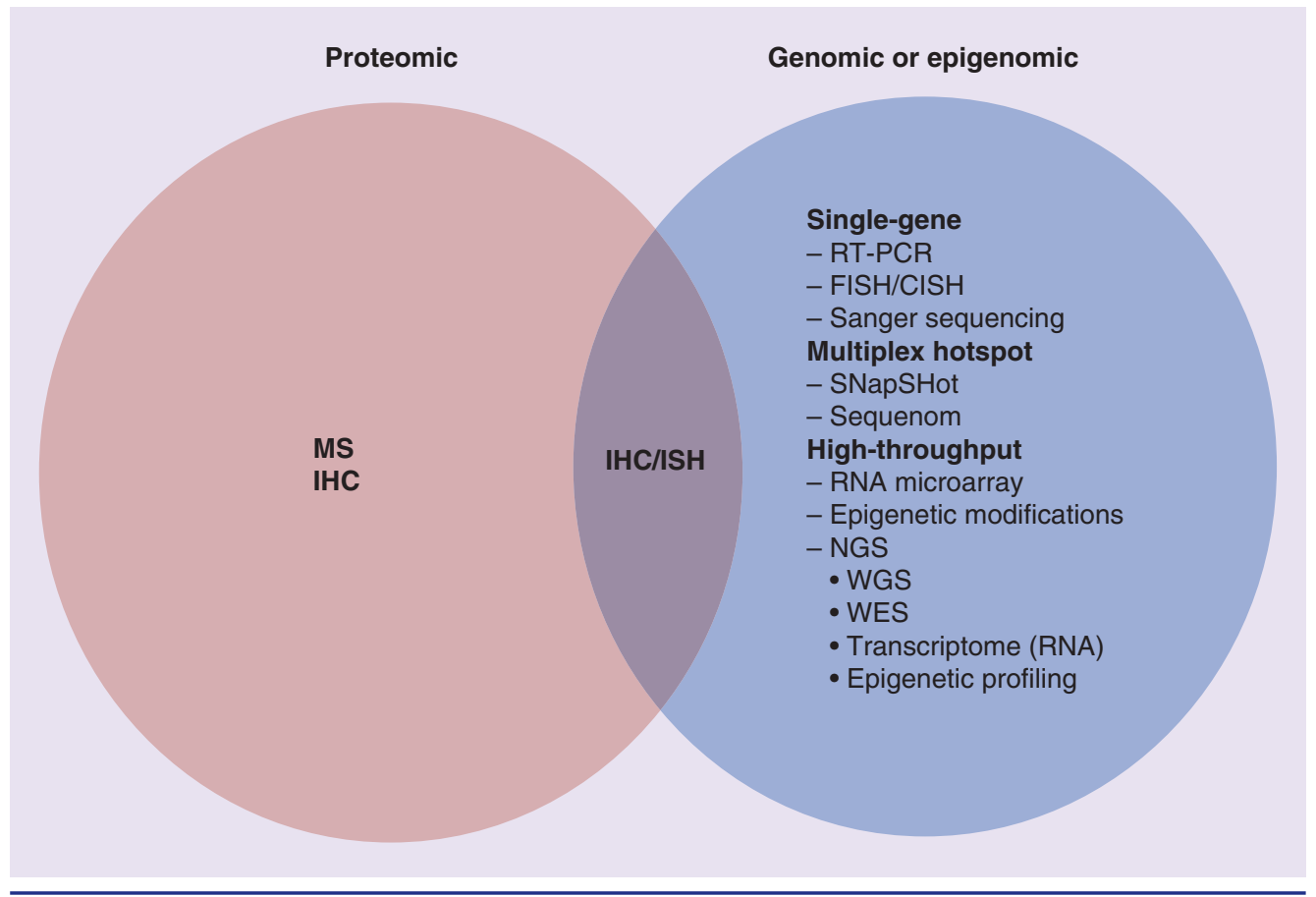

Figure 2. Venn diagram illustrating current proteomic and genomic/epigenomic molecular diagnostic platforms and areas of overlap with immunohistochemistry combination platforms. CISH: Chromogenic in situ hybridization; CNV: Copy number variation; IHC: Immunohistochemistry; MS: Mass spectrometry; NGS: Next-generation sequencing; RT-PCR: Reverse transcription PCR; SNP: Single nucleotide polymorphism; WES: Whole-exome sequencing; WGS: Whole-genome sequencing. 
require that mutant DNA alleles must comprise over $25 \%$ of the total DNA signals [64]. Since samples for mutational analysis may be of limited availability such as small tissue biopsies or cytological specimens (and the high proportion of normal cells that may be present), the low sensitivity of direct sequencing presents critical disadvantages, not least the limited sensitivity that may render some aberrations undetected [65].

Although not yet in routine clinical practice, next-generation sequencing (NGS) platforms have now advanced to the point where several genomes can now be rapidly sequenced in costeffective parallel runs, and with high sensitivity. Massively parallel NGS sequencing technologies can be applied to whole-genome and whole-exome sequencing to detect mutations and polymorphisms, transcriptome sequencing for quantification of gene expression, small RNS sequencing for miRNA profiling, large-scale analysis of DNA methylation and chromatin immunoprecipitation mapping of DNA-protein interaction [66]. Targeted DNA enrichment methods allow even higher genome throughput at a reduced cost per sample, and NGS is thus now being considered by many laboratories for routine diagnostic use in identifying targetable molecular aberrations in lung cancer specimens [65]. Significantly, DNA isolated from fine-needle aspiration (FNA) slides yields comprehensive, accurate and statistically indistinguishable sequence information compared with that obtained from FFPE tissue [67,68]. NGS also affords highly concordant results when comparing primary tumors and metastases from NSCLC patients, and may thus also monitor the molecular evolution of the disease during treatment, and guide targeted treatment decisions upon recurrence [69].

\section{- The role of the pulmonologist, interventional radiologist \& surgeon}

The advent of individualized therapy targeting driver molecular alterations has led to a rapid increase in the number of putative biomarkers that need to be assessed in each patient. Approaches in current development are thus aimed toward introducing comprehensive molecular characterization in standard clinical practice based on minimal tumor biopsy material. However, the routine molecular testing of tumor tissue to guide treatment represents a significant paradigm shift in NSCLC therapy, and will require a standardized collaborative approach to specimen acquisition and processing. Pulmonologists, interventional radiologists or surgeons will need to obtain samples of sufficient quality and quantity for both histologic diagnosis and molecular analysis. Core biopsies are preferred, where four specimens from central lesions may be adequate for diagnosis, but up to six may need to be considered for detailed molecular analysis [70]. For patients with advanced disease where resected tumor samples may not be available, tissue for tumor typing and molecular analysis can be obtained by a number of non-invasive techniques, where optimization of tissue recovery and minimization of procedure-related morbidity are crucial [71]. Sputum cytology is particularly valuable for centrally located tumors, but its sensitivity is location-specific, and the majority of times does not help. Transthoracic needle aspiration (TTNA) is a minimally invasive procedure with excellent sensitivity for peripheral lesions in particular. However, the false-negative rate with TTNA is high, and results may be non-diagnostic; further testing is then required, particularly for early stage disease where resection may be feasible [71]. Flexible bronchoscopy was previously effective for central lesions only, but the recent introduction of endobronchial ultrasound (EBUS) has increased the diagnostic yield to safely include peripheral lesions in a minimally invasive bronchoscopy procedure [71]. EBUS-guided transbronchial needle aspiration (EBUS-TBNA) provides a high diagnostic yield that allows combined pathologic and molecular analysis of metastatic lymph nodes [72]. This method benefits from rapid on-site cytologic examination to confirm adequate samples for both molecular testing and pathologic diagnosis to guide treatment decisions. If the lesion/lymph nodes are close to the esophagus, then endoscopic esophageal ultrasound (EGD/EUS) is performed. Although larger tissue samples are still preferable, NSCLC diagnostics have shifted toward these minimally invasive procedures, and techniques have been developed whereby molecular testing can be performed on smaller amounts of tissue. Completely non-invasive techniques such as analysis of circulating tumor cells are also under investigation [73-75]. There is even evidence of detecting circulating DNA mutations in NSCLC [76].

In the author's clinical practice, if pulmonary or interventional radiology is unable to access the tissue, our surgeons are intimately involved in tumor tissue procurement. It is crucial to have tumor board discussions as to how best to obtain the tissue and process for the various biomarkers. 
Tumor re-biopsy

For patients who have progressed following treatment with standard chemotherapy or EGFR/ALK TKIs, tumor re-biopsy is feasible and may provide information that guides second-line treatment, including the identification of new oncogenic drivers. The choice of technique and material to re-biopsy (lung, nodes, liver etc.) is a key issue [77]. In one study, percutaneous transthoracic lung biopsy with CT guidance provided adequate specimens for mutational analysis in 75 (80\%) of 94 patient re-biopsy specimens [78]. Of 75 specimens, 35 were tested for EGFR mutation, 34 for $A L K$ rearrangement and six for both. The results were positive for sensitizing EGFR mutation (exon 19 or 21) in 20, for EGFR T790M mutation in five and for $A L K$ rearrangement in 11. Postprocedural complications occurred in 13 (14\%) of 94 patients. Re-biopsy may thus provide informative data for targeted treatment decisions [77-79].

\section{- Oncologist \& multidisciplinary team interactions}

Intimate dialog and understanding is required between the medical oncologist and the pulmonologist/interventional radiologist/surgeon, and molecular pathologist, first in making decisions as to whether individual or parallel diagnostic tests are appropriate. The oncologist is likely to lead the initial evaluation, when enrichment strategies for known genetic aberrations might be considered. For example, in patients (particularly female) with adenocarcinoma who are never-to-light smokers and of Asian origin, EGFR mutation testing has been suggested as an appropriate first-line diagnostic test $[80,81]$. On the other hand, $A L K$ rearrangement most commonly occurs in patients with adenocarcinoma who are never-to-light smokers, of younger median age at diagnosis [25,82-83], and who have benefited from chemotherapy for a relatively long period [84]. These patient characteristics could justify $A L K$ rearrangement testing as a first diagnostic approach, however, using this approach to dictate screening and preselection strategy, although potentially improving costeffectiveness of treatment per quality-adjusted life year [85], may also mean some patients who may benefit from approved targeted therapies or could be enrolled in targeted treatment clinical trials are missed. Indeed, a recent report suggests that $A L K$ rearrangement testing would be more effectively performed by selecting just young NSCLC patients without EGFR mutations, whereas selection on the basis of a non-smoking or adenocarcinoma history, as reported in previous studies, may not correctly identify the patient groups with $A L K$-rearranged tumors [86]. For this enrichment strategy, patients would therefore also have to first be tested for EGFR mutations. We however suggest to have more comprehensive parallel genotyping or sequencing that would enable all tumors to be characterized for all potential targetable aberrations, and would include those patients with squamous disease in which fewer targetable molecular aberrations are known but which do occur, particularly in the FGFR and PI3K signaling pathways [29], and not all of whom are currently recommended for EGFR mutation or $A L K$ rearrangement testing [4]. This approach would enable a more rapid initiation of targeted therapy, either with approved single agents, or through enrolment in trials, and would also identify potential combination therapy options when multiple mutations are detected [87]. This strategy may also benefit patients who are smokers, and where a more complex genetic mutation burden is more likely [29].

\section{Conclusion}

The information provided by increasingly complex algorithms comprising data from not only established single diagnostics, but also rapidly advancing multiplexed genotyping or NGS techniques, should be interpreted in a multidisciplinary forum, where actionable results can be clearly identified among potentially large sets of data. Close interactions between the various disciplines within a multimodal team are required to ensure optimum patient diagnostic and treatment flow (Figure 3).

\section{Future perspective}

It may be anticipated that the number of approved targeted therapies for specific molecular subtypes of NSCLC will increase dramatically over the coming decade. In addition to an increasing number and diversity of companion diagnostics, increasingly complex diagnostic algorithms may be required to facilitate an efficient appraisal and molecular diagnosis of a large volume of patients. As newer drugs are identified, it is crucial to have ongoing dialog between the various healthcare providers for the patient. Communication between the pulmonologist/interventional radiologist/surgeon, and molecular pathologist will be important to plan and optimize tumor biopsy tissue acquisition, handling and suitability and ultimately facilitate the identification and complete 


\section{Treatment}

Surgery

Radiotherapy

Chemotherapy

Approved targeted therapy

Targeted therapy clinical trial

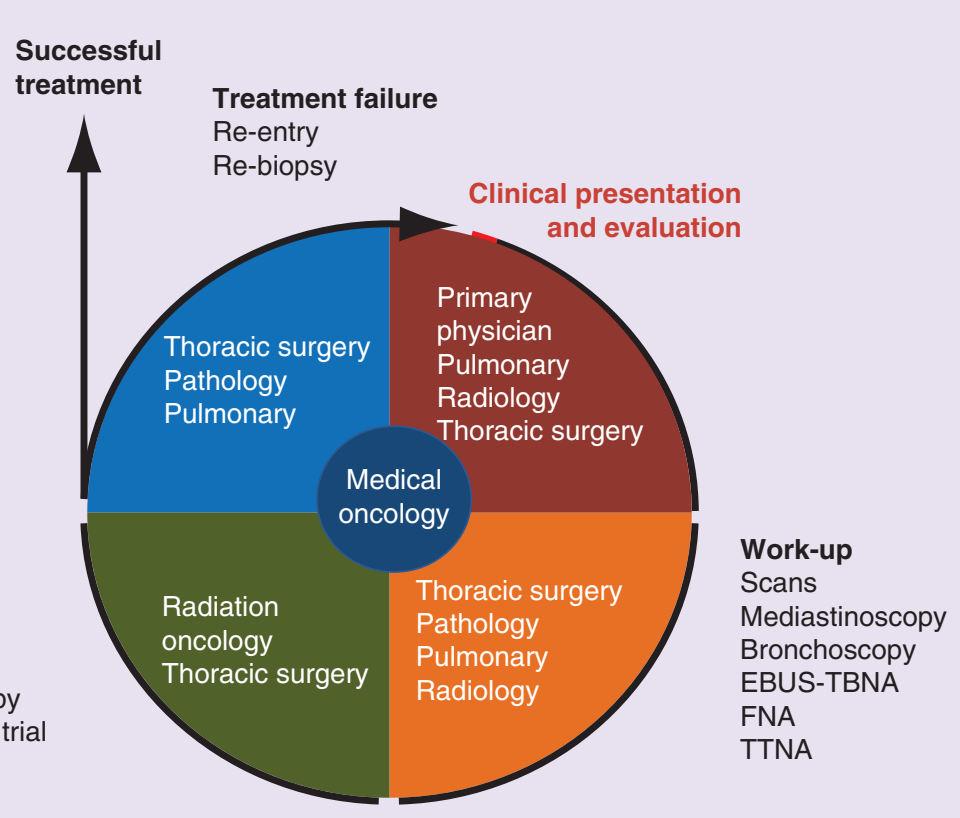

Diagnosis

$\mathrm{FISH} / \mathrm{IHC}$

NGS

Multiplex platforms

Figure 3. Key components, diagnostic flow and interactions of a multidisciplinary tumor board, showing the complete treatment cycle and re-entry for relapsed patients following re-biopsy. EBUS-TBNA: Endobronchial ultrasound-transbronchial needle aspiration; FNA: Fine-needle aspiration; IHC: Immunohistochemistry; NGS: Next-generation sequencing; TTNA: Transthoracic needle aspiration.

classification of the malignancy, subtype and molecular profile. Molecular tumor boards will be important in deciphering potentially complex data, so that actionable data are disseminated and made available to treating physicians. Ultimately, discussion is then required between the medical oncologist, and/or thoracic surgeon and radiation oncologist, including patient consultation, in making precise individual treatment decisions.

As we go forward, it is also crucial to recognize that we need not only molecular identification of the various subsets of lung cancer, but also tumor tissue for various clinical trials. As an example, for anti-PD1 and anti-PDL1 therapeutics, a number of biomarkers have to be analyzed. In order to do this, a certain amount of tumor tissue is needed. It is important to have these discussions with the patient and the coordinating team. We have come a long way for lung cancer, and the future for therapy and survival is optimistic. Although molecularly targeted therapies are not curative for advanced disease, approved agents of this class have already dramatically improved the response to treatment for limited patient subgroups. Molecular diagnostics are not only vital in identifying patients who may benefit from these and newer therapies, but also to identify potential drug targets responsible for inevitable resistance to these agents. With the various biomarkers being identified from even the smallest biopsies, we will have arrived at better therapeutics.

\section{Disclaimer}

The author meets criteria for authorship as recommended by the International Committee of Medical Journal Editors (ICMJE), is fully responsible for all content and editorial decisions and was involved at all stages of manuscript development.

\section{Financial \& competing interests disclosure}

The author has no relevant affliations or financial involvement with any organization or entity with a financial interest in or financial conflict with the subject matter or materials discussed in the manuscript. This includes employment, consultancies, honoraria, stock ownership or options, expert 
testimony, grants or patents received or pending or royalties.

Medical editorial assistance was provided by Matthew Naylor, who was funded by Novartis Pharmaceuticals Corporation.

\section{Open access}

This work is licensed under the Creative Commons

Attribution-NonCommercial 3.0 Unported License. To

view a copy of this license, visit http://creativecommons.org/

licenses/by-nc-nd/3.0/

\section{EXECUTIVE SUMMARY}

\section{Personalized therapy for non-small-cell lung cancer}

- A new approach to the collection and analysis of non-resected, small biopsy samples and cytology is needed to both distinguish histology and detect a growing number of molecular aberrations in non-small-cell lung cancer (NSCLC).

- A personalized approach to NSCLC treatment will maximize the effectiveness of currently available and newly emerging targeted therapies.

- New driver mutations provide therapeutic options for specific patient subgroups, as exemplified by ALK, BRAF and potential new targets such as NTRK1 and RET.

\section{Multidisciplinary approach to an NSCLC molecular therapeutic algorithm: pathologic considerations}

- Current guidelines are insufficient for the selection of patients for clinical trial-based evaluations of new targeted therapies.

- Optimum diagnostics for newer genetic alterations such as ALK rearrangement have not been definitively established, but FISH is the US FDA-approved clinical standard.

- Companion diagnostic platforms, advances in translational genomics and proteomics have now provided the foundation for the rapid advance of the diagnostics and management of NSCLC.

The role of the pulmonologist \& interventional radiologist

- NSCLC diagnostics have shifted toward minimally invasive procedures, whereby molecular testing can be performed on smaller amounts of tissue; optimization of tissue recovery and minimization of procedure-related morbidity are crucial.

- Pulmonologists need to obtain samples of sufficient quality and quantity for both histologic diagnosis and molecular analysis.

- For patients who have progressed following treatment with standard chemotherapy or targeted agents, tumor re-biopsy is feasible and may guide second-line treatment.

\section{Oncologist \& multidisciplinary team interactions}

- Intimate dialog and understanding is required between members of multidisciplinary teams in making decisions on individual or parallel diagnostic tests.

- Parallel genotyping or sequencing enables tumors to be characterized for all potential targetable aberrations, including in squamous disease, and may benefit patients with a more complex genetic mutation burden.

\section{References}

Papers of special note have been highlighted as:

- of interest; $\bullet$ of considerable interest

1 Jemal A, Bray F, Center MM, Ferlay J, Ward E, Forman D. Global cancer statistics. CA Cancer J. Clin. 61(2), 69-90 (2011).

2 Siegel R, Ma J, Zou Z, Jemal A. Cancer statistics, 2014. CA Cancer J. Clin. 64(1), 9-29 (2014).
3 Herbst RS, Heymach JV, Lippman SM. Lung cancer. N. Engl. J. Med. 359(13), 1367-1380 (2008).

4 NCCN Guidelines: NSCLC. v3. (2014). www.nccn.org

5 American Cancer Society. NSCLC: 5-year survival rates (2014). www.cancer.org

6 Langer CJ, Besse B, Gualberto A, Brambilla E, Soria JC. The evolving role of histology in the management of advanced non-small-cell lung cancer. J. Clin. Oncol. 28(36), 5311-5320 (2010).

7 Pineda C, Cadogan KV, Cadogan MA. Distribution of metastases in NSCLC: economic impact of imaging. Presented at: 2009 American Society Clinical Onology Annual Meeting. Orlando, FL, USA, 29 May-2 June 2009 (Abstract e19036). 
8 NCI surveillance epidemiology and end results (2014).

http://seer.cancer.gov

9 Schiller JH, Harrington D, Belani CP et al. Comparison of four chemotherapy regimens for advanced non-small-cell lung cancer. N. Engl. J. Med. 346(2), 92-98 (2002).

10 Sandler A, Gray R, Perry MC et al. Paclitaxel-carboplatin alone or with bevacizumab for non-small-cell lung cancer. N. Engl. J. Med. 355(24), 2542-2550 (2006).

11 Scagliotti GV, Parikh P, von Pawel J et al. Phase III study comparing cisplatin plus gemcitabine with cisplatin plus pemetrexed in chemotherapy-naive patients with advancedstage non-small-cell lung cancer. J. Clin. Oncol. 26(21), 3543-3551 (2008).

12 Fossella F, Pereira JR, von Pawel J et al. Randomized, multinational, Phase III study of docetaxel plus platinum combinations versus vinorelbine plus cisplatin for advanced non-small-cell lung cancer: the TAX 326 study group. J. Clin. Oncol. 21(16), 3016-3024 (2003).

13 Mok TS, Wu YL, Thongprasert S et al. Gefitinib or carboplatin-paclitaxel in pulmonary adenocarcinoma. $N$. Engl. J. Med. 361(10), 947-957 (2009).

14 Mitsudomi T, Morita S, Yatabe Y et al. Gefitinib versus cisplatin plus docetaxel in patients with non-small-cell lung cancer harbouring mutations of the epidermal growth factor receptor (WJTOG3405): an open label, randomised Phase 3 trial. Lancet Oncol. 11(2), 121-128 (2010).

15 Maemondo M, Inoue A, Kobayashi K et al. Gefitinib or chemotherapy for non-small-cell lung cancer with mutated EGFR. N. Engl. J. Med. 362(25), 2380-2388 (2010).

16 Sequist LV, Yang JC, Yamamoto $\mathrm{N}$ et al. Phase III study of afatinib or cisplatin plus pemetrexed in patients with metastatic lung adenocarcinoma with EGFR mutations. J. Clin. Oncol. 31(27), 3327-3334 (2013).

- Phase III study of afatinib in patients with EGFR-mutant non-small-cell lung cancer (NSCLC) upon which approval was based.

17 Rosell R, Carcereny E, Gervais R et al. Erlotinib versus standard chemotherapy as first-line treatment for European patients with advanced EGFR mutation-positive non-smallcell lung cancer (EURTAC): a multicentre, open-label, randomised Phase 3 trial. Lancet Oncol. 13(3), 239-246 (2012).

18 Zhou C, Wu YL, Chen G et al. Erlotinib versus chemotherapy as first-line treatment for patients with advanced EGFR mutation- positive non-small-cell lung cancer (OPTIMAL, CTONG-0802): a multicentre, open-label, randomised, Phase 3 study. Lancet Oncol. 12(8), 735-742 (2011).

19 Shaw AT, Kim DW, Nakagawa K et al. Crizotinib versus chemotherapy in advanced ALK-positive lung cancer. N. Engl. J. Med. 368(25), 2385-2394 (2013).

20 Kwak EL, Bang YJ, Camidge DR et al. Anaplastic lymphoma kinase inhibition in non-small-cell lung cancer. $N$. Engl. J. Med. 363(18), 1693-1703 (2010).

- Phase I trial demonstrating the efficacy of crizotinib in patients with $A L K$-positive NSCLC.

21 Shaw AT, Kim DW, Mehra R et al. Ceritinib in $A L K$-rearranged non-small cell lung cancer. N. Engl. J. Med. 370, 1189-1197 (2014).

- Phase I trial demonstrating the efficacy of ceritinib in patients with $A L K$-positive NSCLC, which formed the basis for regulatory approval.

22 Seto T, Kiura K, Nishio M et al. CH5424802 (RO5424802) for patients with ALKrearranged advanced non-small-cell lung cancer (AF-001JP study): a single-arm, open-label, Phase 1-2 study. Lancet Oncol. 14(7), 590-598 (2013).

23 US FDA. (2014). US FDA Drugs and Approvals Database: Ceritinib. www.fda.gov

24 Chugai Pharmaceutical Co. Ltd. Chugai Pharmaceutical Co. Ltd. New Drug Application. www.chugai-pharm.co.jp

25 Pao W, Girard N. New driver mutations in non-small-cell lung cancer. Lancet Oncol. 12(2), 175-180 (2011).

26 Heist RS, Engelman JA. SnapShot: non-small cell lung cancer. Cancer Cell 21(3), 448.e2 (2012).

27 Vaishnavi A, Capelletti M, Le AT et al. Oncogenic and drug-sensitive NTRK1 rearrangements in lung cancer. $N a t$. Med. 19(11), 1469-1472 (2013).

28 Tsuta K, Kohno T, Yoshida A et al. RETrearranged non-small-cell lung carcinoma: a clinicopathological and molecular analysis. Br. J. Cancer 110(6), 1571-1578 (2014).

29 Heist RS, Sequist LV, Engelman JA. Genetic changes in squamous cell lung cancer: a review. J. Thorac. Oncol. 7(5), 924-933 (2012).

30 Sholl LM, Weremowicz S, Gray SW et al. Combined use of ALK immunohistochemistry and FISH for optimal detection of ALK-rearranged lung adenocarcinomas. J. Thorac. Oncol. 8(3), 322-328 (2013).

31 Dutt A, Ramos AH, Hammerman PS et al. Inhibitor-sensitive FGFR1 amplification in human non-small cell lung cancer. PLoS ONE 6(6), e20351 (2011).

32 Jin G, Kim MJ, Jeon HS et al. PTEN mutations and relationship to $E G F R, E R B B 2$, $K R A S$, and TP53 mutations in non-small cell lung cancers. Lung Cancer 69(3), 279-283 (2010).

33 Spoerke JM, O’Brien C, Huw L et al. Phosphoinositide 3-kinase (PI3K) pathway alterations are associated with histologic subtypes and are predictive of sensitivity to PI3K inhibitors in lung cancer preclinical models. Clin. Cancer Res. 18(24), 6771-6783 (2012).

34 Hammerman PS, Sos ML, Ramos AH et al. Mutations in the DDR2 kinase gene identify a novel therapeutic target in squamous cell lung cancer. Cancer Discov. 1(1), 78-89 (2011).

35 Janku F, Stewart DJ, Kurzrock R. Targeted therapy in non-small-cell lung cancer - is it becoming a reality? Nat. Rev. Clin. Oncol. 7(7), 401-414 (2010).

36 Planchard D, Mazieres J, Riely GJ et al. Interim results of Phase II study BRF113928 of dabrafenib in BRAF V600E mutationpositive non-small cel lung cancer (NSCLC) patients. Presented at: 2013 American Society Clinical Onology Annual Meeting.

Chicago, IL, USA, 31 May-4 June 2013 (Abstract8009).

37 Peters S, Michielin O, Zimmermann S. Dramatic response induced by vemurafenib in a BRAF V600E-mutated lung adenocarcinoma. J. Clin. Oncol. 31(20), e341-e344 (2013).

38 Marchetti A, Felicioni L, Malatesta S et al. Clinical features and outcome of patients with non-small-cell lung cancer harboring $B R A F$ mutations. J. Clin. Oncol. 29(26), 3574-3579 (2011).

39 Paik PK, Arcila ME, Fara M et al. Clinical characteristics of patients with lung adenocarcinomas harboring $B R A F$ mutations. J. Clin. Oncol. 29(15), 2046-2051 (2011).

40 Doebele RC, Vaishnavi A, Capelletti M et al. NTRK1 gene fusions as a novel oncogene target in lung cancer. Presented at: 2013 American Society Clinical Onology Annual Meeting. Chicago, IL, USA, 31 May-4 June 2013 (Abstract 8023).

41 Kris MG, Johnson BE, Berry LD et al. Using multiplexed assays of oncogenic drivers in 
lung cancers to select targeted drugs. JAMA 311(19), 1998-2006 (2014).

42 Sequist LV, Heist RS, Shaw AT et al. Implementing multiplexed genotyping of non-small-cell lung cancers into routine clinical practice. Ann. Oncol. 22(12), 2616-2624 (2011).

-. Demonstrates the potential utility of multiplexed genotyping of NSCLC in clinical practice.

43 Brahmer JR. Harnessing the immune system for the treatment of non-small-cell lung cancer. J. Clin. Oncol. 31(8), 1021-1028 (2013).

44 Peters S, Adjei AA, Gridelli C et al. Metastatic non-small-cell lung cancer (NSCLC): ESMO Clinical Practice Guidelines for diagnosis, treatment and follow-up. Ann. Oncol. 23(Suppl. 7), vii56-vii64 (2012).

45 Travis WD, Brambilla E, Riely GJ. New pathologic classification of lung cancer: relevance for clinical practice and clinical trials. J. Clin. Oncol. 31(8), 992-1001 (2013).

46 Kim MJ, Shin HC, Shin KC, Ro JY. Best immunohistochemical panel in distinguishing adenocarcinoma from squamous cell carcinoma of lung: tissue microarray assay in resected lung cancer specimens. Ann. Diagn. Pathol. 17(1), 85-90 (2013).

47 Lindeman NI, Cagle PT, Beasley MB et al. Molecular testing guideline for selection of lung cancer patients for EGFR and ALK tyrosine kinase inhibitors: guideline from the College of American Pathologists, International Association for the Study of Lung Cancer, and Association for Molecular Pathology. J. Thorac. Oncol. 8(7), 823-859 (2013).

-• Current guidelines for treatment-guiding molecular testing for ALK and EGFR in lung cancer.

48 Fiala O, Pesek M, Finek J, Benesova L, Bortlicek Z, Minarik M. Gene mutations in squamous cell NSCLC: insignificance of EGFR, KRAS and PIK3CA mutations in prediction of EGFR-TKI treatment efficacy. Anticancer Res. 33(4), 1705-1711 (2013).

49 Shiao TH, Chang YL, Yu CJ et al. Epidermal growth factor receptor mutations in small cell lung cancer: a brief report. J. Thorac. Oncol. 6(1), 195-198 (2011).

50 Gruber K, Horn H, Kalla J et al. Detection of rearrangements and transcriptional up-regulation of ALK in FFPE lung cancer specimens using a novel, sensitive, quantitative reverse transcription polymerase chain reaction assay. J. Thorac. Oncol. 9(3), 307-315 (2014).

51 Katayama R, Shaw AT, Khan TM et al. Mechanisms of acquired crizotinib resistance in ALK-rearranged lung Cancers. Sci. Transl. Med. 4(120), 120ra17 (2012).

52 Nitta H, Tsuta K, Yoshida A et al. New methods for ALK status diagnosis in non-small-cell lung cancer: an improved ALK immunohistochemical assay and a new, Brightfield, dual ALK IHC-in situ hybridization assay. J. Thorac. Oncol. 8(8), 1019-1031 (2013).

53 Vincent MD, Kuruvilla MS, Leighl NB, Kamel-Reid S. Biomarkers that currently affect clinical practice: $E G F R, A L K, M E T$, KRAS. Curr. Oncol. 19(Suppl. 1), S33-S44 (2012).

-• Summary of current diagnostic markers that influence clinical decision making in NSCLC.

54 Camidge DR, Skokan M, Kiatsimkul P et al. Native and rearranged ALK copy number and rearranged ALK cell count in NSCLC: Implications for ALK inhibitor therapy. Presented at: 2012 American Society Clinical Onology Annual Meeting. Chicago IL, USA, June 1-5 June 2012 (Abstract 7534).

55 Peled N, Palmer G, Hirsch FR et al. Next-generation sequencing identifies and immunohistochemistry confirms a novel crizotinib-sensitive ALK rearrangement in a patient with metastatic non-small-cell lung cancer. J. Thorac. Oncol. 7(9), e14-e16 (2012).

- Study demonstrating of the importance of next-generation sequencing in identifying patients for targeted treatment.

56 Sun JM, Choi YL, Won JK et al. A dramatic response to crizotinib in a non-small-cell lung cancer patient with IHC-positive and FISH-negative ALK. J. Thorac. Oncol. 7(12), e36-e38 (2012).

57 Wallander ML, Geiersbach KB, Tripp SR, Layfield LJ. Comparison of reverse transcription-polymerase chain reaction, immunohistochemistry, and fluorescence in situ hybridization methodologies for detection of echinoderm microtubule-associated proteinlike 4-anaplastic lymphoma kinase fusion-positive non-small cell lung carcinoma: implications for optimal clinical testing. Arch. Pathol. Lab. Med. 136(7), 796-803 (2012).

58 McLeer-Florin A, Moro-Sibilot D, Melis A et al. Dual IHC and FISH testing for ALK gene rearrangement in lung adenocarcinomas in a routine practice: a French study. J. Thorac. Oncol. 7(2), 348-354 (2012).

59 Thunnissen E, Bubendorf L, Dietel M et al. EML4-ALK testing in non-small cell carcinomas of the lung: a review with recommendations. Virchows Arch. 461(3), 245-257 (2012).
60 Chihara D, Suzuki R. More on crizotinib. $N$. Engl. J. Med. 364(8), 776-777; Author reply 778 (2011).

61 Cabillic F, Gros A, Dugay F et al. Parallel FISH and immunohistochemical studies of ALK Status in 3244 non-small-cell lung cancers reveal major discordances. J. Thorac. Oncol. 9(3), 295-306 (2014).

62 Bar J, Damianovich M, Hout Siloni G et al. Genetic mutation screen in early non-smallcell lung cancer (NSCLC) specimens. Clin. Lung Cancer. 15(2), 159-165 (2014).

63 Huang D, Kim DW, Kotsakis A et al. Multiplexed deep sequencing analysis of ALK kinase domain identifies resistance mutations in relapsed patients following crizotinib treatment. Genomics 102(3), 157-162 (2013).

64 Endo K, Konishi A, Sasaki H et al. Epidermal growth factor receptor gene mutation in non-small cell lung cancer using highly sensitive and fast TaqMan PCR assay. Lung Cancer 50 (3), 375-384 (2005).

65 Meldrum C, Doyle MA, Tothill RW. Next-generation sequencing for cancer diagnostics: a practical perspective. Clin. Biochem. Rev. 32(4), 177-195 (2011).

66 Daniels MG, Bowman RV, Yang IA, Govindan R, Fong KM. An emerging place for lung cancer genomics in 2013. J. Thorac. Dis. 5(Suppl. 5), S491-S497 (2013).

67 Karnes HE, Duncavage EJ, Bernadt CT. Targeted next-generation sequencing using fine-needle aspirates from adenocarcinomas of the lung. Cancer Cytopathol. 122(2 ), 104-113 (2013).

68 Young G, Wang K, He J et al. Clinical next-generation sequencing successfully applied to fine-needle aspirations of pulmonary and pancreatic neoplasms. Cancer Cytopathol. 121(12), 688-694 (2013).

69 Vignot S, Frampton GM, Soria JC et al. Next-generation sequencing reveals high concordance of recurrent somatic alterations between primary tumor and metastases from patients with non-small-cell lung cancer. J. Clin. Oncol. 31(17), 2167-2172 (2013).

70 Ofiara LM, Navasakulpong A, Ezer N, Gonzalez AV. The importance of a satisfactory biopsy for the diagnosis of lung cancer in the era of personalized treatment. Curr. Oncol. 19 (Suppl. 1), S16-S23 (2012).

71 Rivera MP, Mehta AC. American College of Chest Physicians. Initial diagnosis of lung cancer: ACCP evidence-based clinical practice guidelines (2nd edition). Chest 132 (3 Suppl.), 131S-148S (2007).

72 Nakajima T, Yasufuku K. How I do it - optimal methodology for multidirectional 
analysis of endobronchial ultrasound-guided transbronchial needle aspiration samples. J. Thorac. Oncol. 6(1), 203-206 (2011).

73 Maheswaran S, Sequist LV, Nagrath S et al. Detection of mutations in EGFR in circulating lung-cancer cells. $N$. Engl. J. Med. 359(4), 366-377 (2008).

74 Marchesi V. Lung cancer: ALK status of NSCLC reflected in CTCs. Nat. Rev. Clin. Oncol. 10 (7), 366 (2013)

75 Pailler E, Adam J, Barthelemy A et al. Detection of circulating tumor cells harboring a unique ALK rearrangement in ALK-positive non-small-cell lung cancer. J. Clin. Oncol. 31(18), 2273-2281 (2013).

76 Newman AM, Bratman SV, To J et al. An ultrasensitive method for quantitating circulating tumor DNA with broad patient coverage. Nat. Med. 20(5), 548-554 (2014).

77 Dujon C, Chouaid C, Do P et al. Feasibility and clinical impact of re-biopsy in advanced non-small cell lung cancer: a prospective multicentric study in real world setting (GFPC study 12-01). Presented at: 14th World Conference on Lung
Cancer. Amsterdam, The Netherlands, July 3-7 July 2011 (Abstract MO07.09).

78 Yoon HJ, Lee HY, Lee KS et al. Repeat biopsy for mutational analysis of non-small cell lung cancers resistant to previous chemotherapy: adequacy and complications.

Radiology 265(3), 939-948 (2012).

79 Arcila ME, Oxnard GR, Nafa Ket al. Rebiopsy of lung cancer patients with acquired resistance to EGFR inhibitors and enhanced detection of the T790M mutation using a locked nucleic acid-based assay. Clin. Cancer Res. 17(5), 1169-1180 (2011).

80 Ma BB, Hui EP, Mok TS. Population-based differences in treatment outcome following anticancer drug therapies. Lancet Oncol. 11(1), 75-84 (2010).

81 Mitsudomi T, Kosaka T, Yatabe Y. Biological and clinical implications of EGFR mutations in lung cancer. Int. J. Clin. Oncol. 11(3), 190-198 (2006).

82 Wong DW, Leung EL, So KK et al. The $E M L 4-A L K$ fusion gene is involved in various histologic types of lung cancers from nonsmokers with wild-type EGFR and
KRAS. Cancer 115(8), 1723-1733 (2009).

83 Rodig SJ, Mino-Kenudson M, Dacic S et al. Unique clinicopathologic features characterize ALK-rearranged lung adenocarcinoma in the western population. Clin. Cancer Res. 15(16), 5216-5223 (2009).

84 Tufman AL, Edelmann M, Gamarra F et al. Preselection based on clinical characteristics in German non-small-cell lung cancer patients screened for $E M L 4-A L K$ translocation. J. Thorac. Oncol. 9(1), 109-113 (2014).

85 Atherly AJ, Camidge DR. The costeffectiveness of screening lung cancer patients for targeted drug sensitivity markers. $B r$. J. Cancer 106(6), 1100-1106 (2012).

86 Kobayashi M, Sakakibara T, Inoue A et al. An effective enrichment strategy for $E M L 4-A L K$ fusion gene screening in patients with non-small cell lung cancer. Respir. Investig. 52(1), 49-56 (2014).

87 Shames DS, Wistuba II. The evolving genomic classification of lung cancer. J. Pathol. 232(2), 121-133 (2014). 